\title{
CEFALÉIA PÓS-PUNÇÃO SUBARACNÓIDEA: INFLUÊNCIA DO CALIBRE DA AGULHA E DA DEAMBULAÇÃO PRECOCE."
}

\author{
Altamir Perpétuo Ferreira** \\ Laura Regina Parreira Duarte ${ }^{* *}$
}

\begin{abstract}
RESUMO: Buscamos verificar a influência do calibre da agulha e da deambulação na ocorrência de cefaléia pós punção subaracnóidea. A amostra constou de 40 pacientes, divididos em 2 grupos de 20. Um grupo foi submetido a punção subaracnóidea com agulha $80 \times 7$, e o outro com agulha $80 \times 5$. Após a cirurgia os grupos foram redivididos em sub-grupos de 10 pacientes: um sub-grupo recebeu orientação para permanecer no leito por 24 horas pós cirurgia, o outro para deambular após 4 horas. Os resultados demonstraram $100 \%$ de ausência de cefaléia nos pacientes puncionados com agulha $80 \times 5$. Nos pacientes puncionados com agulha $80 \times 7$ apresentaram cefaléia: $30 \%$ dos que deambularam entre 4 e 6 horas e $10 \%$ dos que permaneceram 24 horas no leito.
\end{abstract}

\begin{abstract}
With this research, we verified the influence of needle caliber on the incidence of headaches in patients after subrachonoid puncture. The sample consisted at $\mathbf{4 0}$ patients, divided into two groups of 20 patients. One group was tested with an $80 \times 7$ needle during subrachonoid puncture while the other group was tested with an $80 \times 5$ needle during the procedure. After the surgery each group was redivided into subgroups of 10 patients; this one subgroup from each larger group was instructed to lie flaton their backs for 24 hours after surgery. The other subgroup from each category was instructed to lie down for just 4 hours after surgery and to then get up and walk around. The results demonstrate a total absence of headaches after surgery in patients who were treated with an $80 \times 5$ needle. Of patientes treated with an $80 \times 7$ needle, $30 \%$ of the subgroup which got up after only 4 to 6 hours experienced headaches, while just $10 \%$ of the subgroup which termained laying down for 24 hours expeienced headaches.
\end{abstract}

\section{INTRODUÇÃO}

O que despertou em nós o interesse na realização desta pesquisa foi o fato de fazermos parte da equipe de saúde de um Centro Cirúrgico já há vários anos, e constantemente participarmos de discussões quanto à etiologia da cefaléia pós punção subaracnóidea.

A anestesia subaracnóidea, ou raquianestesia, é descrita por WILDSMITH (9) como mais fácil de aprender, de rápida execução, mais objetiva na identificação do posicionamento da agulha, sua ação tem início mais rápido, e, a difusão é mais controlável, quando comparado ao bloqueio peridural. Além disso, com menor dose de anestésico local, produz-se um bloqueio intenso, que é mais constante na sua distribuição. Por estas razões, a anestesia subaracnóidea é preferida para injeções únicas.

Segundo THORNEBERRY e TOMAS (8), o grande inconveniente da punção subaracnóidea é a possibilidade da cefaléia pós-operatória, caracterizada por aumentar de intensidade na posição ortostática, e ser aliviada com a posição supina. Ela é considerada grave, quando outros sinais e sintomas como náuseas, vômitos, estrabismo, visão borrada, diplopia, zumbidos ou vertigens, ocorrem concomitantemente.

De acordo com LEE e ATKINSON ${ }^{(5)}$ na punção subaracnóidea, a agulha atravessa a dura-mater, que consiste de uma camada fibrosa forte, formando uma

\footnotetext{
* Esta pesquisa foi realizada com a colaboração dos médicos anestesiologistas BELARMINO B. NETTO e CARLOS HUMBERTO, redigido com auxílio de professores da faculdade de Enfermagem e Obstetrícia de Fernandópolis.

** Alunos do $4^{\circ}$ ano de graduação em enfermagem da Faculdade de Enf ermagem e Obstetricia de Fernandópolis - S.P
} 
bainha tubular presa, separada da parede óssea. Suas principais fibras são no sentido longitudinal, o que dificulta o fechamento após a punção, ocorrendo a formação de um sulco pela introdução da agulha, que pode permanecer aberto por vários dias, como mostra uma necrópsia feita após 11 dias da punção.

FLAATTEN e COLS (4) acreditam que o calibre da agulha exerce influência sobre a incidência da cefaléia pós-punção subaracnóidea. MARTIN e COLS (6) não partilham desta opinião, defendem a idéia que o paciente deve ficar em decúbito dorsal por 24 horas, recebendo hidratação abundante.

MIHIC (7) recomenda como fator importante para evitar cefaléia a introdução da agulha com bisel em direção longitudinal em relação as fibras da duramater, sem cortá-las.

COSTA e COLS (2), preconizam a manutenção dos pacientes no pós-operatório imobilizados no leito, sem travesseiros, com generosa hidratação parenteral, ou, com solução hipertônica de cloreto de sódio.

Entretanto, tais condutas têm sido contestadas por outros autores como THORNBERRY e TOMAS (8), BELDO e COLS (1) e FLAATEN e COLS (3). Tais autores acreditam que, tanto a permanência no leito, como a deambulação precoce, não interferem na incidência da cefaléia.

$\mathrm{Na}$ instituição onde foi realizada esta pesquisa, comumente a punção subaracnóidea é realizada com agulha calibre $80 \times 7$ e os pacientes ficam imobilizados em decúbito dorsal por 24 horas pós-cirurgia, recebendo hidratação endovenosa. Embora não dispondo de dados estatísticos, observamos de modo assistemático a existência de cefaléia em muitos destes pacientes. Após discussões constantes com a equipe médica, conseguimos a colaboração de dois anestesiologistas para a realização desta pesquisa, que terá como objetivo verificar a influência do calibre da agulha e deambulação na ocorrência de cefaléia pós punção subaracnóidea.

\section{METODOLOGIA}

A pesquisa foi realizada no centro cirúrgico de um hospital geral de médio porte, filantrópico, no noroeste paulista. Os dados foram coletados no perío- do de maio a julho de 1991 .

A amostra constou de 40 pacientes, com idade de 21 a 55 anos, sendo 11 do sexo masculino e 29 do sexo feminino. Todos os pacientes foram esclarecidos quanto ao objetivo e procedimento da pesquisa, e fizeram parte da amostra somente os que concordaram.

O procedimento constou de duas fases distintas:

a. Os pacientes foram divididos em dois grupos de 20 pacientes cada um, sendo que um grupo foi submetido a punção subaracnóidea com agulha calibre $80 \times 7$ e o outro grupo de 20 pacientes com agulha calibre $80 \times 5$, ambos com punção em sentido longitudinal.

b. Ao final do procedimento cirúrgico os pacientes foram encaminhados para o leito e, procedeu-se a uma nova divisão.

O grupo de 20 pacientes submetidos a punção subaracnóidea com agulha calibre $80 \times 7$, foi dividido em dois sub-grupos de 10 pacientes cada um. Um sub-grupo foi orientado a permanecer no leito, sem travesseiro por 24 horas pós cirurgia, recebendo hidratação endovenosa. Ooutro sub grupo foi orientado a permanecer no leito, sem travesseiro por 4 horas pós cinurgia, e a deambular após este período, desde que suas condições fisicas permitissem, recebendo a mesma hidratação endovenosa que o outro sub-grupo.

O mesmo procedimento se deu com o grupo de 20 pacientes submetidos a punção subaracnóidea com agulha calibre $80 \times 5$.

Todos os pacientes permaneceram três dias sob observação quanto à queixa de cefaléia e outros sintomas associados.

\section{APRESENTAÇÃO E DISCUSSÃO DOS RESULTADOS}

Os dados obtidos são apresentados nas tabelas a seguir. As tabelas le 2 apresentam a distribuição de pacientes segundo o procedimento cinúrgico, enquanto as tabelas 3 e 4 apresentam a incidência de cefaléia segundo o calibre da agulha utilizado e o tempo de permanência no leito. 


\section{ESQUEMA}

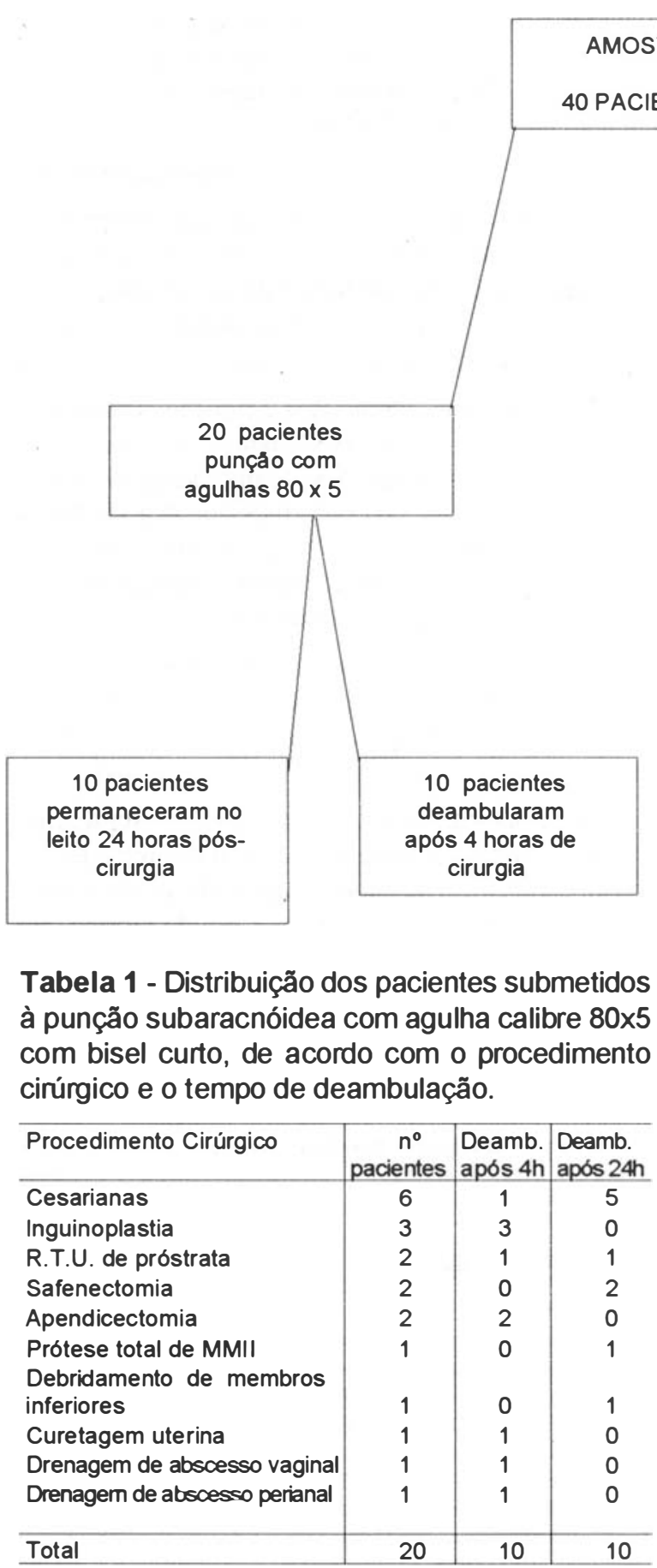

Destes 20 pacientes, 10 foram orientados para deambular 4 horas após a cinurgia. Dentre estes encontram-se os que foram submetidos as seguintes cirurgias: curetagem uterina, drenagem de abscesso vaginal, drenagem de abscesso perianal, inguenoplas- tia, apendictomia, uma cesariana e uma R.T.U de próstata. Os demais receberam orientação para permanecer no leito por 24 horas após cirurgia.

Tabela 2 - Distribuição dos pacientes submetidos a punção subaracnóidea com agulha calibre $80 \times 7$ com bisel curto, de acordo com o procedimento cirúrgico e o tempo de deambulação.

\begin{tabular}{l|c|c|c}
\hline Procedimento Cirúrgico & $\begin{array}{c}\mathrm{n}^{\circ} \\
\text { pacientes }\end{array}$ & $\begin{array}{c}\text { Deamb. } \\
\text { após 4h }\end{array}$ & $\begin{array}{c}\text { Deamb. } \\
\text { após 24h }\end{array}$ \\
\hline Cesarianas & 10 & 4 & 6 \\
Curetagem uterina & 2 & 2 & 0 \\
Hemorroidectomia & 2 & 2 & 0 \\
Ligadura tubária & 2 & 2 & 0 \\
Meniscectomia joelho & 1 & 0 & 1 \\
RTU de próstata & 2 & 0 & 2 \\
Amputaçáo de & & & \\
$\quad$ membro inferior & 1 & 0 & 1 \\
\hline Total & 20 & 10 & 10 \\
\hline
\end{tabular}

Destes 20 pacientes, 10 foram orientados para deambular 4 horas após a cirurgia. Dentre estes encontram-se os que foram submetidos as seguintes cirurgias: curetagem uterina, hemorroidectomia, ligadura tubária, quatro cesarianas. Os demais pacientes 
foram orientados a permanecer no leito por 24 horas após a cirurgia.

Tabela 3 - Incidência de cefaléia de acordo com o calibre da agulha utilizada para a punção subaracnóidea.

\begin{tabular}{c|cc|cc|c}
\hline \multirow{2}{*}{ CEFALÉIA } & \multicolumn{3}{|c|}{ CALIBRE DA AGULHA } & $80 \times 5$ & \multirow{2}{*}{ TOTAL } \\
\cline { 2 - 6 } & $n^{0}$ & $\%$ & $n^{0}$ & $\%$ & \\
\hline Sim & - & - & 04 & 20 & 04 \\
\hline Nåo & 20 & 100 & 16 & 80 & 36 \\
\hline Total & 20 & 100 & 20 & 100 & 40 \\
\hline
\end{tabular}

Observamos nesta tabela que dos pacientes submetidos a punção subaracnóidea com agulha calibre $80 \times 5$, $100 \%$ não apresentou cefaléia; dos pacientes submetidos à punção subaracnóidea com agulha calibre $80 \times 7$, $20 \%$ apresentaram cefaléia e $80 \%$ não apresentaram.

Tabela 4 - Incidência de cefaléia nos pacientes que foram submetidos a punção subaracnóidea com agulha calibre $80 \times 7$, bisel curto, de acordo com o tempo de permanência no leito.

\begin{tabular}{c|cc|rr|r}
\hline \multirow{2}{*}{ CEFALÉIA } & \multicolumn{4}{|c|}{ CALIBRE DA } & \multicolumn{2}{|c|}{} & \multicolumn{2}{|c|}{ TULHA } & $80 \times 7$ & \multirow{2}{*}{ TOTAL } \\
\cline { 2 - 5 } & $n^{0}$ & $\%$ & $n^{0}$ & $\%$ & \\
\hline Sim & 03 & 30 & 01 & 10 & 04 \\
\hline Nåo & 07 & 70 & 09 & 90 & 16 \\
\hline Total & 10 & 100 & 10 & 100 & 20 \\
\hline
\end{tabular}

Observamos nesta tabela que dos pacientes submetidos a punção subaracnóidea com agulha calibre 80x7, que deambularam após 4 a 6 horas de cinurgia, 30\% apresentaram cefaléia. Dos pacientes que permaneceram 24 horas no leito, $10 \%$ apresentaram cefaléia.

Em todos os casos de cefaléia não houve queixa de sintomas tais como visão borrada, diplopia, zumbidos, vertigens, náuseas e outros.
De acordo com LEE e ATKINSON (5) cerca de $40 \%$ dos pacientes apresentam cefaléia pós-punção subaracnóidea. Esta porcentagem se eleva acima de $80 \%$ naqueles pacientes que já apresentam tendência a cefaléia. Estes autores não fazem diferenciação quanto ao calibre da agulha.

A partir deste estudo nos foi possível concluir que:

Dos pacientes submetidos à punção subaracnóidea com agulha calibre $80 \times 5,100 \%$ não apresentou cefaléia, não havendo portanto, diferença entre os que permaneceram no leito por 24 horas pós cinurgia e os que deambularam após 4 a 6 horas.

Dos pacientes submetidos a punção subaracnóidea com agulha calibre $80 \times 7$, houve diferença entre os sub grupos em relação ao tempo de permanência no leito, sendo que dos pacientes que deambularam após 4 horas de cirurgia, 30\% apresentou cefaléia, e, dos pacientes que permaneceram no leito por 24 horas pós cinurgia $10 \%$ apresentaram cefaléia.

Diante destes resultados, a equipe de saúde que participou desta pesquisa, acredita que a melhor conduta é proceder a punção subaracnóidea com a agulha calibre $80 \times 5$ com bisel curto, sempre que possível.

Salientamos porém que este trabalho representa o início de uma investigação maior, pois pretendemos continuar a coleta de dados, ampliando assim a nossa amostra. Continuamos também o estudo teórico, em busca de outras variáveis importantes.

A coleta de dados para a ampliação da amostra, será realizada em outros hospitais da região, pois na instituição onde se realizou esta pesquisa, a equipe de anestesiologista passou a utilizar a agulha calibre $80 \times 5 \mathrm{em}$ todos os casos possíveis.

\section{REFERÊNCIAS BIBLIOGRÁFICAS}

1. BELDO, C.N. e COLS. Cefaléia pós requianestesia: importância do decúbito no pós operatório. Rev. Bras. deAnestesia. v. 35, p. 7-11, 1985.

2. COSTA, D.A.A., GOMES, J.A., NETO, P.C., MATTA, M.J., Profilaxia da cefaléia pós punção de dura-mater com agulha de peridural pelo uso de solução hipertônica a $1,5 \%$. Rev. Bras. de Anestesia, v. 36, p. 37-43, 1986.

3. FLAATEN, H., RODT, S., ROSLAND, J., VAMNES, J., Postoperative headache in young patients after spinal anesthesia. Anaesthesia, v. 42, p. 202-205, 1987.

4. FLAATTEN, H., RODT, S.A., VAMNES, J., ROSLAND, J., WISBORG, T., KOLLER, M.W., Post dural puncture headache. A comparison between 26-and-29 gauge needls in young patientes. Anaesthesia, v. 44, p. 147-149, 1989.

5. LEE, J.A., ATKINSON, R.S., Manual de anestesiologia. Trad. de Milton Marques da Luz. Rio de Janeiro: Atheneu, 1976.
6. _ Anestesia: Teoria, Técnica, Prática. Trad. de Milton Marques da Luz. Rio de Janeiro: Atheneu, 1984.

7. MARTIN, Y.N., GUIAVARC'H, M., OLIVIER, J., DUCROT, P. , Role du calibre $22 \mathrm{G}$ et $25 \mathrm{G}$ de l'aiguille dans l'apparition des céphalées aprés rachianesthésie. Cahiers d'anesthesiol, v. 35, p. 535-537, 1987.

8. MIHIC, D.N., Post spinal headache and relationship of needle bevel to longitudinal dural fibers. Regional Anesth. v. 10, p. 76-81, 1985 .

9. THORNEBERRY, E.A., TOMAS, T.A., Posture and post-spinal headache a controlled trial in 80 obstetric patients. $\mathrm{Br}$. J. Anaesth., v. 60, p. 195-197, 1988.

10. WILDSMITH, J.AW., Intrathecal o estradural: witch approach surgery? Br. J. Anaesth., v. 59, p. 397-398, 1987. 